\title{
EXPERIMENTS IN SQUARE LATTICE WITH A COMMON TREATMENT IN ALL BLOCKS*
}

\author{
F. PIMENTEL GOMES ** \\ GLAUCO P. ViEGAS***
}

\section{SUMMARY}

This paper deals with a generalization of square lattice designs, with $\mathrm{k}^{2}$ treatments in blocks of $\mathrm{k}+1$ plots, the extra plot in each block receiving a standard treatment, the same for all blocks. The new design leads to lower variances for contrasts between adjusted treatment means

\section{INTRODUCTION}

Since a long time ago, the Instituto Agronômico de Campinas, a research institute located in Campinas, São Paulo, Brasil, uses for its experiments with corn (maize) varieties and hybrias, square lattices with $\mathrm{k}^{2}$ treatments and blocks of $k+1$ plots, the extra plot in each block receiving a standard variety or hybrid, the same for all blocks, not included among the $\mathrm{k}^{2}$ original treatments. It is clear, therefore, that these square lattice experiments include, on the whole, $\mathrm{k}^{2}+1$ treatments in blocks of $\mathrm{k}+1$ plots. For example, in a $3^{2}$ lattice, with 2 orthogonal replications, and treatments $1,2, \ldots, 9$, plus treatment A (standard variety), the blocks would be as follows:

\section{Block 1: $123 \mathrm{~A}$ \\ Block 2: $456 \mathrm{~A}$ \\ Block 3: $789 \mathrm{~A}$ \\ Block 4: $147 \mathrm{~A}$ \\ Block 5: $258 \mathrm{~A}$ \\ Block 6: $369 \mathrm{~A}$}

This paper deals with the intrablock analysis of these designs.

\section{INTRABLOCK ANALYSIS}

In these designs we have $v=k^{2}+1$ treatments in a square lattice with $\mathbf{m}$ orthogonal replications, $b=m k$ blocks, of $k+1$ plots. There are $\mathrm{k}^{2}$ regular treatments $(1,2, \ldots$, $\mathrm{k}^{2}$ ), plus a common treatment $\mathrm{A}$. The parameter $\lambda_{\mathrm{ij}}$ is equal to 1 for regular treatments

* Received for publication in 21/11/1975.

* Department of Mathematics and Statistics, ESALQ/USP.

*** Fundação Cargill. 
that appear in the same block (first associates), equal to zero for regular treatments that do not appear in the same block (second associates). But when one of the treatments in the common one, the $\lambda_{\mathrm{ij}}=\mathrm{m}$. $\mathrm{S}_{\mathrm{o}}$, the normal equations (KEMPTHORNE, 1952; PIMENTEL GOMES, 1969) will have coefficients

$$
\begin{aligned}
& c_{\mathrm{ii}}=\mathrm{m}\left(1-\frac{1}{\mathrm{k}+1}\right)=\frac{\mathrm{mk}}{\mathrm{k}+1}, \\
& \mathrm{c}_{\mathrm{ij}}=-\frac{1}{\mathrm{k}+1} \text { (first associates), } \\
& c_{\mathrm{ij}}=0 \text { (second associates) } \\
& c_{\mathrm{iA}}=\frac{\mathrm{m}}{\mathrm{k}+1} \quad(\text { a regular treatment and the common treatment), } \\
& c_{\mathrm{AA}}=\mathrm{mk}\left(1-\frac{1}{\mathrm{k}+1}\right)=\frac{\mathrm{mk}^{2}}{\mathrm{k}+1}
\end{aligned}
$$

For the example above, the equation corresponding to treatment $\mathbf{1}$ is:

$$
\frac{m k}{k+1} t_{1}-\frac{1}{k+1} t_{2}-\frac{1}{k+1} t_{3}-\frac{1}{k+1} t_{4}-\frac{k 1}{k+1} t_{7}-\frac{1}{k+1} t_{A}=Q_{1}
$$

and for treatment $\mathrm{A}$ (common) it is:

$$
-\frac{m}{k+1} t_{1} \cdot \frac{m}{k+1} t_{2} \cdot \ldots \cdot \frac{m}{k+1} t_{9}+\frac{m^{2}}{k+1} t_{A}=Q_{A} \cdot
$$

Since matrix $\mathrm{C}=\left(\mathrm{c}_{\mathrm{ij}}\right)$ is singular, we introduce a restriction for the treatments effects, which can be

$$
\sum_{\mathrm{i}=1}^{\mathrm{k}^{2}} \mathrm{t}_{\mathrm{i}}+\mathrm{kt}_{\mathrm{A}}=0 .
$$

The solution of the system of normal equation gives then:

$$
\begin{gathered}
\hat{\mathrm{t}}_{\mathrm{A}}=\frac{1}{\mathrm{mk}} \mathrm{Q}_{\mathrm{A}}, \\
\hat{\mathrm{t}}_{\mathrm{i}}=\frac{1}{\mathrm{~m}} \mathrm{Q}_{\mathrm{i}}+\frac{1}{\mathrm{k}(\mathrm{mk}-\mathrm{k}+\mathrm{m})} \mathrm{Q}_{\mathrm{A}}+\frac{1}{\mathrm{~m}(\mathrm{mk}-\mathrm{k}+\mathrm{m})}\left[\mathrm{S}_{1}\left(\mathrm{Q}_{\mathrm{i}}\right)+\mathrm{S}_{2}\left(\mathrm{Q}_{\mathrm{i}}\right)+\ldots+\mathrm{S}_{\mathrm{m}}\left(\mathrm{Q}_{\mathrm{i}}\right)\right],
\end{gathered}
$$


where $S_{j}\left(Q_{i}\right)$ is the sum of $Q$ 's in the $j^{\text {th }}$ replication, in the block where the $i^{\text {th }}$ treatment appears.

The sum of squares for treatments (adjusted) is:

$$
\text { SST (adjusted) }=\frac{1}{m} \Sigma Q_{i}^{2}+\frac{m \cdot 1}{m(m k-k+1)} Q_{A}^{2}+\frac{1}{m(m k \cdot k+m)} \sum_{j, j}, S_{j j}^{2},(Q),
$$

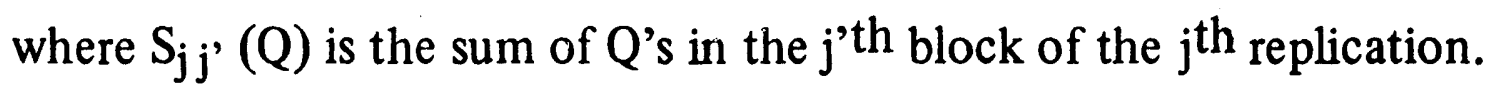

So the analysis of variance is obtained as explained in table 1 .

TABLE 1 - Analysis of variance.

\begin{tabular}{lcl}
\hline Source of variation & D.F. & S.S. \\
\hline Replications & $\mathrm{m}-1$ & As usual \\
Blocks within replications (unadjusted) & $\mathrm{m}(\mathrm{k}-1)$ & As usual \\
Treatments (adjusted) & $\mathrm{k}^{2}$ & By formula \\
Residual & By subtraction & By subtraction \\
\hline Total & $\mathrm{mk}(\mathrm{k}+1)-1$ & As usual \\
\hline
\end{tabular}

The adjusted treatment means are:

$$
\hat{\mathrm{m}}_{\mathrm{i}}=\frac{\mathrm{G}}{\mathrm{mk}(\mathrm{k}+1)}+\hat{\mathrm{t}}_{\mathrm{i}} \quad, \quad \hat{\mathrm{m}}_{\mathrm{A}}=\frac{\mathrm{G}}{\mathrm{mk}(\mathrm{k}+1)}+\hat{\mathrm{t}}_{\mathrm{A}}
$$

where $\mathrm{G}$ is the grand total of all plots.

\section{CONTRASTS BETWEEN TREATMENT MEANS}

There are 3 cases to be studied.

1st associates: Two regular treatments occurring in the same block, for instance, treatments 1 and 2 in the example above:

$$
V\left(\hat{\mathrm{m}}_{\mathrm{i}}-\hat{\mathrm{m}}_{\mathrm{j}}\right)=\frac{2 \sigma^{2}}{\mathrm{~m}}\left[1+\frac{\mathrm{m}-1}{\mathrm{mk}-\mathrm{k}+\mathrm{m}}\right]
$$

2nd associates: Two regular treatments which do not occur in the same block, for instance treatments 1 and 6 in the example above:

$$
\mathrm{V}\left(\hat{\mathrm{m}}_{\mathrm{i}}-\hat{\mathrm{m}}_{\mathrm{j}}\right)=\frac{2 \sigma^{2}}{\mathrm{~m}}\left[1+\frac{\mathrm{m}}{\mathrm{mk}-\mathrm{k}+\mathrm{m}}\right]
$$


3rd associates: A regular treatment and the common treatment:

$$
\mathrm{V}\left(\hat{\mathrm{m}}_{\mathrm{i}}-\hat{\mathrm{m}}_{\mathrm{A}}\right)=\sigma^{2}\left[\frac{1}{\mathrm{~m}}+\frac{1}{\mathrm{mk}}+\frac{\mathrm{k}-1}{\mathrm{k}(\mathrm{mk} \cdot \mathrm{k}+\mathrm{m})}\right]
$$

\section{EXAMPLE OF ANALYSIS}

We shall take as example a $5 \times 5$ square lattice with 4 orthoganal replications and a common treatment A present in all blocks. The experiment was carried out with corn (maize), and harvest expressed in $\mathrm{kg} / \mathrm{ha}$ (Table 2).

For treatments $1,2, \ldots, 25$ and $A$ we compute now the totals $T_{i}(i=1,2, \ldots$, $25, \mathrm{~A})$ and the adjusted treatment totals

$$
Q_{i}=T_{i}-\Sigma_{j} \frac{n_{i j}}{k+1} B_{j}
$$

where $\mathrm{N}=\left(\mathrm{n}_{\mathrm{ij}}\right)$ is the incidence matrix, and $\mathrm{B}_{\mathrm{j}}$ is the total of block $\mathrm{j}$. It is known that the incidence matrix is obtained from elements $n_{i j}$, with $n_{i j}=1$ if treatment $i$ occurs in block $\mathbf{j}$, and $n_{i j}=0$, if it does not occur.

However, it is easier to calculate $Q_{i}^{\prime}=(k+1) Q_{i}$, as done in Table 3 . We have:

$$
\begin{gathered}
T_{1}=22,194 \\
Q_{1}^{\prime}=6 Q_{1}=6 T_{1}-\left(B_{4}+B_{6}+B_{11}+B_{17}\right)= \\
=6 \times 22,194-(35,150+37,957+34,078+33,002)=7,023 \\
Q_{A}^{\prime}=6 Q_{A}=6 \times 113,169-678,476=538 .
\end{gathered}
$$


TABLE 2 - Yields, in $\mathrm{kg} / \mathrm{ha}$, or corn (maize) in the quadruple square lat tice, with a common treatment in all blocks, used as example.

\begin{tabular}{|c|c|c|c|c|c|c|c|}
\hline $\begin{array}{c}\text { Block } \\
\text { No }\end{array}$ & & & & & & & $\begin{array}{l}\text { Block } \\
\text { Totals }\end{array}$ \\
\hline \multicolumn{8}{|c|}{1 st replicate } \\
\hline 1 & $6,126(5)$ & $6,497(11)$ & $6,309(8)$ & $6,271(19)$ & $5,743(22)$ & $6,602(\mathrm{~A})$ & 37,548 \\
\hline 2 & 6,809 (2) & $6,642(10)$ & $5,111(13)$ & $4,646(24)$ & $5,240(16)$ & $6,173(\mathrm{~A})$ & 34,621 \\
\hline 3 & $3,670(4)$ & $6,899(7)$ & $5,770(21)$ & $4,167(18)$ & $4,195(15)$ & $6,430(\mathrm{~A})$ & 31,131 \\
\hline 4 & $6,610(12)$ & $7,166(20)$ & $5,925(23)$ & $5,332(1)$ & $5,509(9)$ & $4,608(\mathrm{~A})$ & 35,150 \\
\hline \multirow[t]{2}{*}{5} & $6,175(14)$ & $7,413(3)$ & $5,768(6)$ & $6,059(17)$ & $5,704(25)$ & 7,202 (A) & 38,321 \\
\hline & & & & & & & 176,771 \\
\hline
\end{tabular}

$2^{\text {nd }}$ replicate

\begin{tabular}{rlllllll}
6 & $6,218(1)$ & $6,692(18)$ & $6,621(10)$ & $6,321(14)$ & $6,318(22)$ & $5,787(\mathrm{~A})$ & 37,957 \\
7 & $5,580(2)$ & $5,586(15)$ & $4,682(23)$ & $6,155(19)$ & $8,237(6)$ & $5,487(\mathrm{~A})$ & 35,727 \\
8 & $6,199(25)$ & $3,844(4)$ & $5,549(12)$ & $5,550(16)$ & $6,480(8)$ & $4,844(\mathrm{~A})$ & 32,466 \\
9 & $5,960(11)$ & $5,019(24)$ & $6,300(7)$ & $6,993(20)$ & $5,996(3)$ & $6,280(\mathrm{~A})$ & 36,548 \\
10 & $6,986(17)$ & $5,191(9)$ & $7,204(5)$ & $6,999(21)$ & $6,394(13)$ & $6,001(\mathrm{~A})$ & 38,775 \\
\hline & & & & & & 181,473
\end{tabular}

$3^{\text {rt }}$ replicate

\begin{tabular}{llllllll}
11 & $6,350(25)$ & $6,519(1)$ & $5,195(13)$ & $6,187(19)$ & $5,412(7)$ & $4,415(\mathrm{~A})$ & 34,078 \\
12 & $4,542(8)$ & $4,330(20)$ & $5,580(2)$ & $3,847(21)$ & $4,339(14)$ & $5,273(\mathrm{~A})$ & 27,911 \\
13 & $4,491(4)$ & $6,285(17)$ & $4,927(11)$ & $3,998(23)$ & $3,846(10)$ & $6,044(\mathrm{~A})$ & 29,591 \\
14 & $5,374(22)$ & $5,690(15)$ & $4,230(3)$ & $4,177(9)$ & $5,416(16)$ & $5,165(\mathrm{~A})$ & 30,052 \\
15 & $6,064(6)$ & $5,992(18)$ & $5,780(5)$ & $5,102(24)$ & $4,692(12)$ & $4,887(\mathrm{~A})$ & 31,817 \\
\hline & & & & & & 153,449
\end{tabular}

$4^{\text {th }}$ replicate

\begin{tabular}{llllllll}
16 & $6,052(7)$ & $6,439(6)$ & $6,600(10)$ & $5,855(8)$ & $7,160(9)$ & $6,687(\mathrm{~A})$ & 38,793 \\
17 & $4,125(1)$ & $5,822(2)$ & $2,956(4)$ & $7,022(5)$ & $7,804(3)$ & $5,273(\mathrm{~A})$ & 33,002 \\
18 & $4,235(13)$ & $4,867(11)$ & $4,734(15)$ & $6,342(14)$ & $7,691(12)$ & $5,744(\mathrm{~A})$ & 33,613 \\
19 & $5,199(16)$ & $3,985(20)$ & $5,029(19)$ & $4,998(18)$ & $6,223(17)$ & $4,823(\mathrm{~A})$ & 30,257 \\
20 & $5,129(29)$ & $5,880(25)$ & $3,609(24)$ & $5,718(23)$ & $5,538(22)$ & $6,044(\mathrm{~A})$ & 31,118 \\
\hline
\end{tabular}

Formulas given in section 2 can be easily changed to use $Q^{\prime}=$ values instead of $\mathrm{Q}$ - values. We obtain:

$$
\hat{\mathrm{t}}_{\mathrm{A}}=\frac{1}{\mathrm{mk}(\mathrm{k}+1)} \mathrm{Q}_{\mathrm{A}}^{\prime}=(1 / 120) 538=4.5 \approx 4
$$




$$
\begin{gathered}
\hat{\mathrm{t}}_{1}=\frac{1}{\mathrm{~m}(\mathrm{k}+1)} \mathrm{Q}_{\mathrm{j}}^{\prime}+\frac{1}{\mathrm{k}(\mathrm{k}+1)(\mathrm{mk}-\mathrm{k}+\mathrm{m})} \mathrm{Q}_{\mathrm{A}}^{\prime}+ \\
+\frac{1}{(\mathrm{k}+1) \mathrm{m}(\mathrm{mk}-\mathrm{k}+\mathrm{m})}\left[\mathrm{S}_{1}\left(\mathrm{Q}_{1}^{\prime}\right)+\mathrm{S}_{2}\left(\mathrm{Q}_{1}^{\prime}\right)+\ldots+\mathrm{S}_{\mathrm{m}}\left(\mathrm{Q}_{1}^{\prime}\right)\right]= \\
=(1 / 24)(-7,023)+(1 / 570)(538)+(1 / 456)(-20,403)=-336 .
\end{gathered}
$$

\begin{tabular}{|c|c|c|c|c|c|c|c|}
\hline $\begin{array}{c}\text { Treat. } \\
\text { no }\end{array}$ & $\begin{array}{l}1^{\text {st }} \\
\text { rep. }\end{array}$ & $\begin{array}{l}2^{\text {nd }} \\
\text { rep. }\end{array}$ & $\begin{array}{l}3^{\text {rd }} \\
\text { rep. }\end{array}$ & $\begin{array}{l}4^{\text {th }} \\
\text { rep. }\end{array}$ & $\mathrm{T}_{\mathrm{i}}$ & $Q_{i}^{\prime}$ & $\mathrm{m}_{\mathrm{i}}$ \\
\hline 1 & 5,332 & 6,218 & 6,519 & 4,125 & 22.194 & - 7,023 & 5,318 \\
\hline 2 & 6,809 & 5,580 & 5,580 & 5,822 & 23.791 & 11,485 & 6,146 \\
\hline 3 & 7,413 & 5,996 & 4,230 & 7,804 & 25.443 & 14,735 & 6,369 \\
\hline 4 & 3,670 & 3,844 & 4,491 & 2,956 & 14.961 & $-36,424$ & 3,948 \\
\hline 5 & 6,126 & 7,204 & 5,780 & 7,022 & 26.132 & 15,650 & 6,406 \\
\hline 6 & 5,768 & 8,237 & 6,064 & 6,439 & 26.508 & 14,390 & 6,466 \\
\hline 7 & 6,899 & 6,300 & 5,412 & 6,052 & 24.663 & 7,428 & 5,899 \\
\hline 8 & 6,309 & 6,480 & 4,542 & 5,855 & 23.186 & 2,398 & 5,846 \\
\hline 9 & 5,509 & 5,191 & 4,177 & 7,160 & 22.037 & $-10,548$ & 5,241 \\
\hline 10 & 6,642 & 6,621 & 3,846 & 6,600 & 23.709 & 1,292 & 5,606 \\
\hline 11 & 6,497 & 5,960 & 4,927 & 4,867 & 22.251 & $-3,794$ & 5,440 \\
\hline 12 & 6,610 & 5,549 & 4,692 & 7,691 & 24.542 & 14,206 & 6,211 \\
\hline 13 & 5,111 & 6,394 & 5,195 & 4,235 & 20.935 & $-15,477$ & 4,927 \\
\hline 14 & 6,175 & 6,321 & 4,339 & 6,342 & 23.177 & 1,260 & 5,829 \\
\hline 15 & 4,195 & 5,586 & 5,690 & 4,734 & 20.205 & $-9,293$ & 5,175 \\
\hline 16 & 5,240 & 5,550 & 5,416 & 5,199 & 21.405 & 1,034 & 5,660 \\
\hline 17 & 6,059 & 6,986 & 6,285 & 6,223 & 25.553 & 16,374 & 6,451 \\
\hline 18 & 4,167 & 6,692 & 5,892 & 4,998 & 21.749 & - 668 & 5,636 \\
\hline 19 & 6,271 & 6,155 & 6,187 & 5,029 & 23.642 & 4,242 & 5,941 \\
\hline 20 & 7,166 & 6,993 & 4,330 & 3,985 & 22.474 & 4,978 & 5,948 \\
\hline 21 & 5,770 & 6,999 & 3,847 & 5,129 & 21.745 & 1,535 & 5,642 \\
\hline 22 & 5,743 & 6,318 & 5,374 & 5,538 & 22.973 & 1,163 & 5,673 \\
\hline 23 & 5,925 & 4,682 & 3,998 & 5,718 & 20.323 & - 9,648 & 5,130 \\
\hline 24 & 4,646 & 5,019 & 5,102 & 3,609 & 18.376 & $-23,848$ & 4,589 \\
\hline 25 & 5,704 & 6,199 & 6,350 & 5,080 & 23.333 & 4,015 & 5,827 \\
\hline \multirow[t]{6}{*}{$\mathbf{A}$} & 6,602 & 5,787 & 4,415 & 6,687 & & & \\
\hline & 6,173 & 5,487 & 5,273 & 5,273 & & & \\
\hline & 6,430 & 4,844 & 6,044 & 5,744 & & & \\
\hline & 4,608 & 6,280 & 5,165 & 4,823 & & & \\
\hline & 7,202 & 6,001 & 4,287 & 6,044 & 113,169 & 538 & 5,658 \\
\hline & 6,771 & 181,473 & 153,449 & $66 ; 783$ & & & \\
\hline
\end{tabular}

TABLE 3 - Data analized, with values of $T_{i}, Q_{i}$ and $m_{i}$ for each treatment. 
On the other hand, we have:

$$
\begin{aligned}
& \hat{\mathrm{m}}_{1}=5,654-336=5,318 \\
& \hat{\mathrm{m}}_{\mathrm{A}}=5,654+4=5,658 .
\end{aligned}
$$

The sum of squares for treatment (adjusted) is:

$$
\begin{aligned}
\mathrm{SST}(\text { adjusted }) & =\frac{1}{\mathrm{~m}(\mathrm{k}+1)^{2}} \Sigma \mathrm{Q}_{\mathrm{i}}^{, 2}+\frac{\mathrm{m} \cdot 1}{\mathrm{~m}(\mathrm{k}+1)^{2}(\mathrm{mk} \cdot \mathrm{k}+\mathrm{m})} \mathrm{Q}_{\mathrm{A}}^{, 2}+ \\
& +\frac{1}{\mathrm{~m}(\mathrm{k}+1)^{2}(\mathrm{mk}-\mathrm{k}+\mathrm{m})} \mathrm{i}_{\mathrm{j}}, \mathrm{S}_{\mathrm{jj}}^{2},\left(\mathrm{Q}_{\mathrm{i})}^{\prime}\right.
\end{aligned}
$$$$
\text { SST }(\text { adjusted })=(1 / 144) 3,888,633,692+(1 / 912) 289,444+
$$$$
+(1 / 2,736) 9,235,411,576=30,380,234
$$

The analysis of variance obtained is given in Table 4.

TABLE 4 - Analy sis of variance of data in table 2.

\begin{tabular}{lcrrr}
\hline Source of variation & D.F. & S.S. & M.S. & F \\
\hline Blocks & 19 & $35,779,509$ & & \\
Treatments (adjusted) & 25 & $30,380,234$ & $1,215,209$ & $1.67^{*}$ \\
Error & 75 & $54,631,221$ & 728,416 & \\
\hline
\end{tabular}

For first assuciates we have:

$$
\hat{\mathrm{V}}\left(\hat{\mathrm{m}}_{\mathrm{i}}-\hat{\mathrm{m}}_{\mathrm{j}}\right)=\frac{2(728,416)}{4}(1+3 / 19)=(0.5789) 728,416=421,680
$$

For second associates the estimate of variance is:

$$
\hat{V}\left(\hat{m}_{i}-\hat{m}_{u}\right)=\frac{2(728,416)}{4}(1+4 / 19)=(0.6053) 728,416=440,910
$$

Finally, for a contrast between the common treatment and any other treatment we have:

$$
V\left(\hat{m}_{i}-\hat{m}_{A}\right)=728,416\left(\frac{1}{4}+\frac{1}{20}+\frac{1}{76}\right)=247,278
$$


For the usual $5 \times 5$ square lattice we should obtain:

$$
\hat{V}\left(\hat{m}_{i}-\hat{m}_{u}\right)=0.6000 s^{2}
$$

for first associates, and

$$
V\left(\hat{m}_{i}-\hat{m}_{u}\right)=0.6333 \mathrm{~s}^{2}
$$

for second associates. We conclude, therefore, that the new design gives lower estimates for these variances.

\section{RESUMO}

O Instituto Agronômico de Campinas vem, há muitos anos, utilizando, nos seus ensaios de milho, reticulados quadrados com $\mathrm{k}^{2}$ tratamentos em blocos de $\mathrm{k}+1$ parcelas, sendo a parcela extra de cada bloco cultivada com um cultivar padrão (variedade ou híbrido), não incluído entre os $\mathrm{k}^{2}$ tratamentos originais. Conclui-se, pois, que esses delineamentos incluem $\mathrm{k}^{2}+1$ tratamentos, em blocos de $\mathrm{k}+1$ parcelas.

$\mathrm{O}$ presente trabalho deduz fórmulas para a análise da variância desses delineamentos, e para a estimação das médias ajustadas de tratamentos. Fórmulas para a variância de diversos contrastes são deduzidas. Finalmente, apresenta-se um exemplo, detalhadamente analisado, de urn ensaio em reticulado quadrado com $\mathrm{k}^{2}=25$, e 4 repetições ortogonais, instalado corn 26 cultivares, em blocos de 6 parcelas.

\section{LITERATURE CITED}

KEMPTHORNE, O., 1952. The Design and Analysis of Experiments. John Wiley \& Sons, New York.

PIMENTEL GOMES, F., 1968. The Solution of Normal Equations of Experiments in Incomplete Blocks. Ciência e Cultura, 20:733-746. 